\title{
Takayasu's Arteritis associated with Tuberculosis Infections
}

\author{
Reshkova V, Kalinova D and Rashkov R \\ Clinic of Rheumatology, Medical University of Sofia, Sofia, Bulgaria
}

Corresponding author: Dr. Valentina Reshkova, Clinic of Rheumatology, Medical University of Sofia, 13 Urvich Str, 1612, Sofia, Bulgaria, Tel: Tel: 359878622443; E-mail: v_reshkova@abv.bg

Received: May 05, 2016; Accepted: Jun 07, 2016; Published: Jun 10, 2016

\section{Abstract}

Takayasu's arteritis (TA) is an inflammatory disease of unknown etiology characterized by granulomatous vasculitis affecting the aorta, its main branches and the pulmonary arteries. It occurs most often in women of child-bearing age. At the time of diagnosis $10 \%$ to $20 \%$ of patients with TA are clinically asymptomatic. The remaining $80 \%$ to $90 \%$ of patients present with systemic or vascular symptoms. The most important points in diagnosing Takayasu's arteritis are the clinical features, physical examination and diagnostic imaging (catheterdirected dye arteriography, magnetic resonance angiography, computed tomographic angiography). The etiology of TA is not clear. A possible relationship between TA and tuberculosis has been suggested. Some studies suggest cross-reaction between Mycobacterium tuberculosis and human heat shock protein. It has been speculated the role of mycobacterial super antigens, which cause polyclonal $\mathrm{T}$ cell activation and massive cytokine release, that induce vascular damage. The use of tuberculostatic drugs is rationale during the treatment of TA. We report two interesting clinical cases of Takayasu's arteritis and tuberculosis. The first clinical case presents a rare case of TA and tuberculous lymphadenitis in an 18 year old male patient. The second case presented TA associated with latent tuberculosis in a 36 year old man.

Keywords: Takayasu's arteritis; Tuberculosis; Granulomatous vasculitis

\section{Introduction}

Takayasu's arteritis (TA) is a granulomatous vasculitis of unknown etiology that affects the aorta, its main branches, and the pulmonary arteries [1]. A possible relationship between TA and tuberculosis has been suggested. Both diseases show similar pathological changes in the form of granulomas on the arterial walls [2,3]. We report two cases of Takayasu's arteritis and tuberculosis.

\section{Case 1}

An 18-year-old male patient presented with complaints of astheno-adynamic syndrome, weight loss, night sweats, dyspnea and fever to $38.5^{\circ} \mathrm{C}$. His symptoms started with low grade fever which used to appear at evening and gradually became persistent.

Physical examination revealed asthenic habit, enlarged peripheral lymph nodes in the left axillary region-firm in consistency, non-tender and not fixed with surrounding structures. It was found pulse celer of the a. radialis dextra, diminished pulse of the a. radialis sinistra and the a. brachialis sinistra. Carotid pulses were presented without bruits. Blood pressure measurements: in the right upper limb 120/80 $\mathrm{mmHg}$, in the left upper limb $80 / 50 \mathrm{mmHg}$. Cardiac examination showed diastolic heart murmur over the right second intercostal space, systolic fremisman over 2 nd and 3 rd left intercostal space, systolic murmur over the left sternal border. Abdomen examinations revealed no abnormal findings. Laboratory findings showed normal white blood cells with predominant lymphocytosis, increased erythrocytes sedimentation rate $(50 \mathrm{~mm}), \mathrm{C}$-reactive protein $63 \mathrm{~g} / \mathrm{l}$ (normal $<6 \mathrm{~g} / \mathrm{l}$ ) and anemia. Liver function test, renal function test and lipids profile were within normal limits.

Chest radiography findings were enlarged hilar lymph nodes, moderate cardiomegaly and dilatation of the ascendent aorta. Echocardiography showed annuloaortic ectasia with aortic valvular regurgitation III-IV grade, dilatation of the left ventricle and systolic dysfunction. Duplex vascular sonograpy of the a.a carotides revealed bilateral concentric thickening wall. Computed tomographic angiography of the aorta and the branches showed concentric thickening of the wall of the aortic arch and the ascending aorta (Figure 1); two concentric stenosis $50-60 \%$ in the proximal and intermediate segment of the a. carotis communis dextra and concentric stenosis in the proximal segment of the a. carotis communis sinistra (Figure 2).

Through enlarge lymph nodes Mantoux test for TB was performed and it was positive $(19 \mathrm{~mm})$, as well as following Quantiferon test. Histopathological report of the axillar lymph node biopsy showed caseating tubercules suggesting tuberculous lymphadenitis.

It was diagnosed Takayasu's arteritis associated with tuberculous lymphadenitis. Combination therapy Methylprednisolone $20 \mathrm{mg} /$ daily and tuberculostatic drugs (Isoniazid, Rifampicin, Pyrazinamide and Etambutol) was started in the patient. 


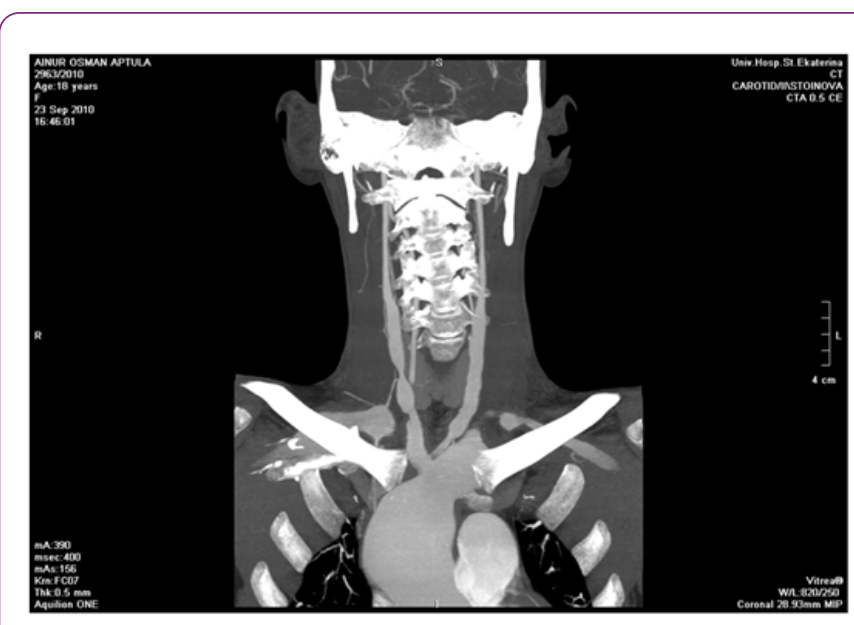

Figure 1 Concentric thickening of the wall of the aortic arch and the ascending aorta.

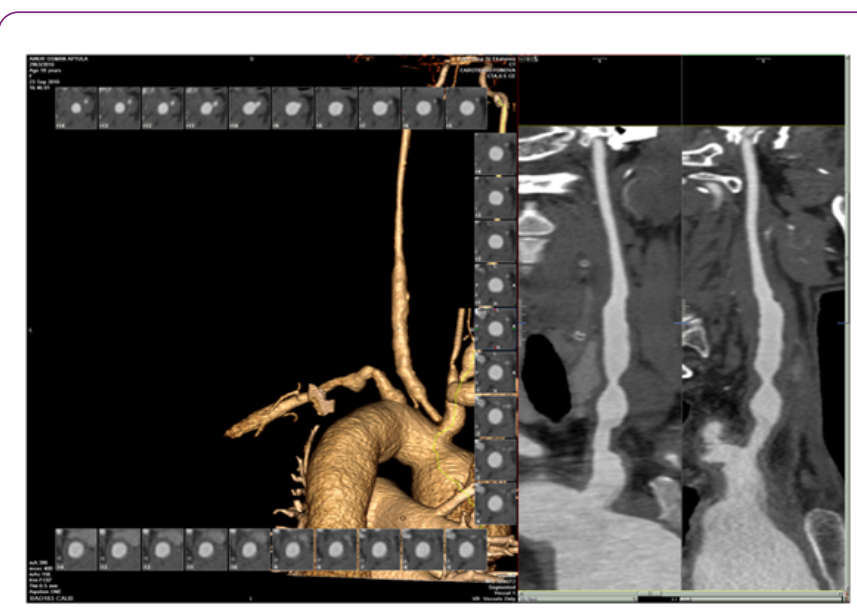

Figure 2 Two concentric stenosis $50-60 \%$ in the proximal and intermediate segment of the a. carotis communis dextra and concentric stenosis in the proximal segment of the a. carotis communis sinistra.

\section{Case 2}

A 36-year old male patient presented with tOC, dyspnea, weight loss, palpitations, and heart oppression.

Physical examination showed normal breath sounds, pulsations of the four limbs were present, peripheral lymph nodes were not enlarged. Cardiac examination showed systolic heart murmur over the broad area of the precordium, propagating to the pulmonary valve diastolic heart murmur over the left sternal border. Abdomen examination revealed no abnormal findings

Laboratory investigations: leukocytosis, hemoglobin was $10.9 \mathrm{gm} / \mathrm{dl}$, erythrocytes sedimentation rate was $70 \mathrm{~mm}$ and C-reactive protein $75.8 \mathrm{~g} / \mathrm{l}$ (normal $<6 \mathrm{~g} / \mathrm{l}$ ). Serologic study for ANA (IFI), ANCA (IFI) was negative. Liver function test, renal function test and lipids profile were within normal limits.
Electrocardiography presented sinus tachycardia with left axis deviation and signs of left ventricle enlargement. Echocardiography showed aortic valvular regurgitation grade III, pulmonary hypertension (35 $\mathrm{mmHg}$ ). Duplex vascular ultrasound revealed significantly narrowed of the aa. carotides communes. Computed tomographic angiography of the aorta and the main branches showed cuff-like thickened wall of the thoracic aorta; occlusion of the a. pulmonalis dextra in proximal segment, as perfusion carried out aa. bronchiales (Figure 3); stenosis of the ostium of the a. coronaris dextra. The angiographic findings weren't observed in the abdominal aorta.

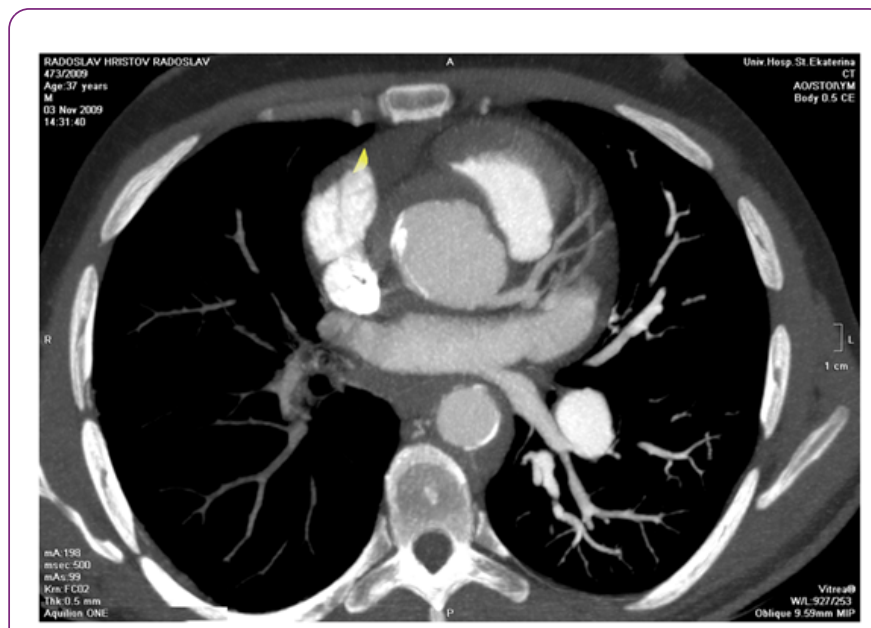

Figure 3 Occlusion of the a. pulmonalis dextra, as perfusion carried out aa. bronchiales.

Therapy with Methylprednisolone $20 \mathrm{mg} /$ daily, Isoniazide $300 \mathrm{mg} /$ daily and antihypertensive drugs (Amlodipine $5 \mathrm{mg}$ / daily; Ramipril $2 \times 5 \mathrm{mg}$; Bisoprolol $5 \mathrm{mg}$ ) was started in the patient.

\section{Discussion}

Takayasu's arteritis is an inflammatory disease of unknown etiology characterized by granulomatous vasculitis affecting the aorta, its main branches, and the pulmonary arteries. It occurs most often in women of child-bearing age [1]. At the time of diagnosis $10 \%$ to $20 \%$ of patients with TA are clinically asymptomatic. The remaining $80 \%$ to $90 \%$ of patients present as a result of symptoms that are systemic or vascular [4]. Systemic symptoms include fatigue, malaise, weight loss, night sweats, fever, arthralgias, or myalgias. Vascular features can be completely asymptomatic or present as transient ischemic attacks, stroke, dizziness, syncope, headache, or visual changes $[1,5,6]$. We present Takayasu's arteritis in two young male patients. Involvement of the vessels in the first described clinical case is completely asymptomatic and the disease being detected incidentally by abnormal vascular finding on examination-diminished pulses of the a. radialis sinistra et al. brachialis sisnistra and asymmetric blood pressure. The presenting clinical symptoms in the second patient are dyspnea, palpitations and heart oppression. Cardiac involvement is related to aortic valvular regurgitation and 
stenosis of the a. coronaris dextra. Aortic regurgitation, result from dilatation of the aortic root is the most frequent cardiac manifestation. Coronary vessels stenosis may develop up to $25 \%$ of patients with TA [7]. Involvement of the pulmonary artery was diagnosed too, but it isn't clinical apparent. By arteriographic studies, pulmonary artery disease may occur in $50 \%$ to $86 \%$ of patients $[8,9]$.

Takayasu's arteritis is one of the first vasculitides to be associated with a specific infectious agents $[2,3,10,11]$. There were performed tests for tuberculosis in two patients with Takayasu's arteritis. The tuberculin test was positive and caseating granulomatous lesions were found in axillar lymph node in the first patients. These findings were suggestive of an active TB, respectively association between specific infection and the vascular process. In the other case the tuberculin skin test was negative, but the following QuantiFERON-TB Gold was positive. QuantiFERON-TB Gold is a test for screening latent tuberculosis [12] through it was diagnosed latent tuberculosis associated with Takayasu's arteritis. Despite the association with tuberculosis and the similarity between lesions in TA and tuberculosis (lymphoplasmatic infiltrate with granuloma formation and giant cells involving the media and adventia), the exact role of Mycobacterium tuberculosis in the pathogenesis of TA is still unknown. Previous studies suggest the cross-reactivity between mycobacteria and a human heat shock protein (HSP) might have a key role. This hypothesis is further supported by the increased expression of HSP in the involved vessels as wells as the activation of subpopulations of T cells $[10,11,13]$. It has also been speculated that $M$. tuberculosis can be triggering factor through its production of superantigens. The super antigens cause polyclonal $\mathrm{T}$ cell activation and massive cytokine release that induce vascular damage [3].

Diagnostic imaging is fundamental to the diagnosis of Takayasu's arteritis and plays an essential role in disease monitoring. Arteriography using catheter-directed intravascular injection of contrast dye, magnetic resonance angiography (MRA) or computed tomographic angiography (CTA) provides information on vascular luminal dimensions [1]. MRA and CTA are safe and noninvasive techniques for assessing vessel patency in Takayasu's arteritis and play an essential role in disease monitoring $[14,15]$. We observed similar patterns of vessel involvement in the described clinical cases, with lesions of the thoracic aorta (ascending aorta, aortic arch) and her branches-carotid arteries, and pulmonary artery in the second case. Complete imaging of the aorta and its main branches should be performed at the time of diagnosis, because such studies can detect clinically occult vascular disease $[14,15]$. CTA will be perform at 6 -month to monitor for the development of new vascular lesions.

\section{References}

1. Hochberg $M$, Silman A, Smolen J, Weinblatt $M$, Weisman $M$ (2011) Takayasu's arteritis. Rheumatology (5thedn) ELSEVIER 1567-1571.

2. Kinare SG (1970) Aortitis in early life in India and its association with tuberculosis. J Pathol 100: 69.

3. Al-Aghbari K, Al-Motarreb A, Et Askar F (2010) Takayasu's arteritis associated with tuberculosis in a young yemeni woman. Heart Views 11: 117-120.

4. Kerr GS, Hallahan CW, Giordano J (1994) Takayasu arteritis. Ann Intern Med 120: 919-929.

5. Sharma BK, Jain S, Sagar S (1996) Systemic manifestation of Takayasu arteritis: the expanding spectrum. Int J Cardiol 54: S149-S154.

6. Sharma BK, Jain S, Suri S (1996) Diagnostic criteria for Takayasu arteritis. Int J Cardiol 54: S141-S147.

7. Park YB, Hong SK, Choi KJ (1992) Takayasu arteritis in Korea: clinical and angiographic features. Heart Vessels Suppl 7: 55-59.

8. Zheng D, Fan D, Lui L (1992) Takayasu arteritis in China: a report of 530 cases. Heart Vessels Suppl 7: 32-36.

9. Jain S, Kumari S, Ganguly NK (1996) Current status of Takayasu arteritis in India. Int J Cardiol 54: S111-S116.

10. Aggarwal A, Chag M, Sinha N, Naik S (1996) Takayasu's arteritis: a role of Mycobacterium tuberculosis and its 65-kDa heat shock protein. Int J Cardiol 55: 49-55.

11. Seko J, Minota S, Kawasaki A (1994) Perforin-secreting killer cell infiltration and expression of a $65-\mathrm{kDa}$ heat shock protein in aortic tissue of patients with Takayasu's arteritis. J Clin Invest 93: 750-758.

12. Manuel O, Kumar D (2008) QuantifERON-TB Gold assay for the diagnosis of latent tuberculosis infection. Expert rev Mol Diagn 8: 247-256.

13. Chaudan SK, Singh M, Nityanand S (2007) Reactivity of gamma/ delta $T$ cells to human $60-k d$ heat-shock protein and their cytotoxicity to aortic endothelial cells in Takayasu arteritis. Arthritis Rheum 56: 2798-2802.

14. Hata A, Noda M, Moriwaki R (1996) Angiographic findings of Takayasu arteritis: New classification. Int J Cardiol 54: S155S163.

15. Tso E, Flamm SD, White RD (2002) Takayasu arteritis: utility and limitations of magnetic resonance imaging in diagnosis and treatment. Arthritis Rheum 46: 1634-1642. 